\title{
Absorption cross section of canonical acoustic holes
}

\author{
Luís C. B. Crispino* and Ednilton S. Oliveira ${ }^{\dagger}$ \\ Faculdade de Física, Universidade Federal do Pará, 66075-110, Belém, PA, Brazil
}

George E. A. Matsas

Instituto de Física Teórica, Universidade Estadual Paulista, Rua Pamplona 145, 01405-900, São Paulo, SP, Brazil

(Received 1 August 2007; revised manuscript received 21 October 2007; published 29 November 2007)

We compute numerically the absorption cross section of a canonical acoustic hole for sound waves with arbitrary frequencies. Our outputs are in full agreement with the expected low- and high-frequency limits.

DOI: 10.1103/PhysRevD.76.107502

PACS numbers: 04.70.-s, 47.35.Rs

It has been more than 25 years since it was first realized by Unruh [1] that the same arguments that lead to Hawking radiation also predict the emission of a thermal spectrum of phonons from sonic holes. This new result has opened a whole research branch, which has been significantly developed since then (see, e.g., Ref. [2] and references therein). In addition, the computation of black hole absorption cross sections has attracted much attention since the seventies (see, e.g., Refs. [3,4]) up to now (see, e.g., Refs. $[5,6])$. For instance, it was only very recently that the absorption cross section for the electromagnetic field in the spacetime of a Schwarzschild black hole has been computed for arbitrary frequencies [7]. Here, we compute numerically the absorption cross section for sound waves with arbitrary frequencies in the spacetime of a canonical acoustic hole [8].

The canonical acoustic hole is described by the line element

$$
d s_{a}^{2}=f(r) d t^{2}-[f(r)]^{-1} d r^{2}-r^{2}\left(d \theta^{2}+\sin ^{2} \theta d \phi^{2}\right),
$$

where $f(r)=1-r_{H}^{4} / r^{4}$. This is a spherically symmetric static spacetime experienced by sound waves in a perfect irrotational barotropic fluid with constant energy density and sound velocity. Here $r_{H}$ is the radius corresponding to the sonic event horizon, i.e. the boundary of the no-return region for phonons, where the radial velocity of the inward fluid equals the sound velocity.

The Klein-Gordon equation, $\square \Psi=0$, can be written in the spacetime (1) as

$$
\frac{1}{f} \frac{\partial^{2} \Psi}{\partial t^{2}}-\frac{1}{r^{2}} \frac{\partial}{\partial r}\left(f r^{2} \frac{\partial \Psi}{\partial r}\right)+\frac{1}{r^{2}} \tilde{\nabla}^{2} \Psi=0 .
$$

Here $\tilde{\nabla}^{2}=\tilde{\eta}^{i j} \tilde{\nabla}_{i} \tilde{\nabla}_{j}$, where $i$ and $j$ denote angular variables in the unit 2-sphere $S^{2}$ with metric $\tilde{\eta}_{i j}$ and inverse metric $\tilde{\eta}^{i j}$ [with signature $(--)$ ].

\footnotetext{
*crispino@ufpa.br

matsas@ift.unesp.br

‡ednilton@ufpa.br
}

The positive-frequency solutions of Eq. (2) can be cast in the form

$$
u_{\omega l m}=\left(\psi_{\omega l}(r) / r\right) Y_{l m}(\theta, \phi) e^{-i \omega t}
$$

with $Y_{l m}(\theta, \phi)$ being the scalar spherical harmonics and $l$ and $m$ being the corresponding angular momentum quantum numbers. In the present case, the functions $\psi_{\omega l}(r)$ satisfy the differential equation

$$
f \frac{d}{d r}\left(f \frac{d \psi_{\omega l}}{d r}\right)+\left[\omega^{2}-V_{a}(r)\right] \psi_{\omega l}=0
$$

with the following scattering potential:

$$
V_{a}(r)=f\left(4 r_{H}^{4} / r^{6}+l(l+1) / r^{2}\right)
$$

We plot $V_{a}(r)$ in Fig. 1, where we can see that it goes to zero as $r \rightarrow r_{H}$ and $r \rightarrow \infty$. In terms of the Wheeler-type coordinate $x=x(r)$ defined by $d / d x \equiv f d / d r$, Eq. (4) can be rewritten as

$$
d^{2} \psi_{\omega l} / d x^{2}+\left[\omega^{2}-V_{a}(x)\right] \psi_{\omega l}=0 .
$$

In order to orthonormalize the solutions of this differential equation, we use the Klein-Gordon inner product [9]

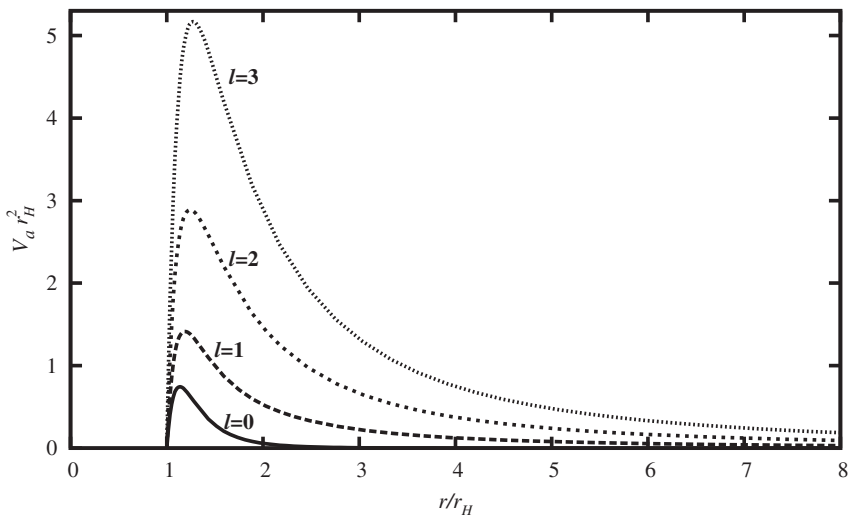

FIG. 1. The scattering potential $V_{a}$ [defined in Eq. (5)] is plotted for different values of the angular momentum $l$. We can see that it vanishes at the canonical acoustic hole horizon $\left(r=r_{H}\right)$. As $r \rightarrow \infty, V_{a}$ falls as $1 / r^{6}$ for $l=0$ and as $1 / r^{2}$ for $l>0$. 


$$
\sigma_{K G}(\phi, \psi)=\int_{\Sigma^{(3)}} d \Sigma^{(3)} n^{\mu} J_{\mu}(\phi, \psi)
$$

with the conserved current defined as

$$
J_{\mu}(\phi, \psi) \equiv i\left[\phi^{*}\left(\nabla_{\mu} \psi\right)-\left(\nabla_{\mu} \phi^{*}\right) \psi\right] .
$$

Here $d \Sigma^{(3)}=\sqrt{-\eta^{(3)}} d^{3} x$ is the invariant volume element of a Cauchy surface $\Sigma^{(3)}, n^{\mu}$ is the future pointing unit vector orthogonal to $\Sigma^{(3)}$, and $\eta^{(3)}$ is the determinant of the metric restricted to $\Sigma^{(3)}$. We impose that

$$
\begin{aligned}
& \sigma_{K G}\left(u_{\omega l m}, u_{\omega^{\prime} l^{\prime} m^{\prime}}\right)=\delta_{l l^{\prime}} \delta_{m m^{\prime}} \delta\left(\omega-\omega^{\prime}\right), \\
& \sigma_{K G}\left(u_{\omega l m}^{*}, u_{\omega^{\prime} l^{\prime} m^{\prime}}\right)=0 .
\end{aligned}
$$

The asymptotic forms of the modes incoming from the past infinity can be written as

$$
\psi_{\omega l}(r) \approx \begin{cases}A_{\omega l} T_{\omega l} e^{-i \omega x} & \left(r \approx r_{H}\right), \\ A_{\omega l} \omega x\left[(-i)^{l+1} h_{l}^{(1) *}(\omega x)+i^{l+1} R_{\omega l} h_{l}^{1}(\omega x)\right] & \left(r \gg r_{H}\right) .\end{cases}
$$

Here $h_{l}^{(1)}(x)$ are the spherical Bessel functions of the third kind [10], $A_{\omega l}$ are normalization constants, $\left|R_{\omega l}\right|^{2}$ and $\left|T_{\omega l}\right|^{2}$ are the reflexion and transmission coefficients, respectively, satisfying the usual probability conservation equation $\left|R_{\omega l}\right|^{2}+\left|T_{\omega l}\right|^{2}=1$. (For a study of the reflection coefficient for superresonant scattering of acoustic disturbances from a rotating acoustic hole in the low-frequency regime see [11].) Note that

$$
\begin{aligned}
& h_{l}^{(1)}(x)=j_{l}(x)+i n_{l}(x) \approx(-i)^{(l+1)} e^{i x} / x \\
& \quad(|x| \gg l(l+1) / 2) .
\end{aligned}
$$

Hence, we obtain from Eq. (7) that

$$
\psi_{\omega l}(r) \approx A_{\omega l}\left(e^{-i \omega x}+R_{\omega l} e^{i \omega x}\right)
$$

in the region $\omega x \gg l(l+1) / 2$.

The absorption cross section is given by

$$
\sigma=-\mathcal{F} / J_{I},
$$

where $\mathcal{F}$ is the total absorbed wave flux and $J_{I}$ is the incident current density.

Since the spacetime of the canonical acoustic hole is asymptotically flat, we consider an incident plane wave propagating in the $z$ direction,

$$
v\left(x^{\mu}\right)=C e^{i \omega(z-t)},
$$

where $C$ is a normalization constant. The incident current density associated with the plane wave (10) is

$$
J_{I}=2|C|^{2} \omega .
$$

In order to find the flux of the absorbed wave, we first expand the plane wave (10) in terms of Legendre functions as [12]

$$
v\left(x^{\mu}\right)=C \sum_{l=0}^{\infty} i^{l}(2 l+1) j_{l}(\omega r) \mathrm{P}_{l}(\cos \theta) e^{-i \omega t},
$$

where $\mathrm{P}_{l}(\cos \theta)$ are the Legendre polynomials and $j_{l}(\omega r)$ are the spherical Bessel functions of the first kind [10]. By using the asymptotic form of $j_{l}(\omega r)$ :

$$
j_{l}(x) \approx \frac{1}{x} \sin \left(x-\frac{l \pi}{2}\right)=\frac{i^{l+1}}{2 x}\left[e^{-i x}-(-1)^{l} e^{i x}\right],
$$

which is valid for $x \gg l(l+1) / 2$, we can write the plane wave (10) asymptotically as

$$
\begin{aligned}
v\left(x^{\mu}\right) \approx & i C \sum_{l=0}^{\infty} \frac{(-1)^{l}(2 l+1)}{2 \omega} \frac{\left[e^{-i \omega r}-(-1)^{l} e^{i \omega r}\right]}{r} \\
& \times \mathrm{P}_{l}(\cos \theta) e^{-i \omega t} .
\end{aligned}
$$

In the case of the canonical acoustic hole the asymptotic form of the incident plane wave will be given by

$$
\begin{aligned}
v_{a}\left(x^{\mu}\right) \approx & i C \sum_{l=0}^{\infty} \frac{(-1)^{l}(2 l+1)}{2 \omega} \frac{\left(e^{-i \omega r}+R_{\omega l} e^{i \omega r}\right)}{r} \\
& \times \mathrm{P}_{l}(\cos \theta) e^{-i \omega t} .
\end{aligned}
$$

Equation (12) can indeed be obtained, up to a global multiplicative constant, by substituting Eq. (8) in Eq. (3), for the case of an incident wave in the $z$ direction.

The flux associated with the mode (8) is

$$
\mathcal{F}=\oint_{r \rightarrow \infty} d \Omega r^{2} J^{r}\left(v_{a}, v_{a}\right)
$$

Using Eqs. (6) and (12), and that

$$
\int_{0}^{\pi} \mathrm{P}_{l}(\cos \theta) \mathrm{P}_{l^{\prime}}(\cos \theta) \sin \theta d \theta=\frac{2}{2 l+1} \delta_{l l^{\prime}},
$$

we obtain

$$
\mathcal{F}=-\frac{2 \pi|C|^{2}}{\omega} \sum_{l=0}^{\infty}(2 l+1)\left(1-\left|R_{\omega l}\right|^{2}\right) .
$$

Now, from Eqs. (9), (11), and (13), we obtain the total absorption cross section for the canonical acoustic hole:

$$
\sigma_{a}=\sum_{l=0}^{\infty} \sigma_{a}^{(l)}=\frac{\pi}{\omega^{2}} \sum_{l=0}^{\infty}(2 l+1)\left|T_{\omega l}\right|^{2} .
$$

In Fig. 2 we plot our results for the partial absorption cross section $\sigma_{a}^{(l)}$ for $l=0$ up to $l=6$. The numerical method used is the same as described in Ref. [13]. We see that the $s$ wave $(l=0)$ contribution is responsible for the nonvanishing cross section in the zero-energy limit. Moreover, for each value of $l>0$, the corresponding partial absorption cross section starts from zero, reaches a 




FIG. 2. The partial absorption cross section $\sigma_{a}^{(l)}$ is plotted as a function of the wave frequency $\omega$, for $l=0$ up to $l=6$. Note that the larger $l$ is, the smaller is the corresponding maximum of $\sigma_{a}^{(l)}$.

maximum value $\sigma_{a}^{(l) \max }$ and decreases asymptotically. We also see that the larger the value of $l$ is (i) the smaller is the corresponding value of $\sigma_{a}^{(l) \max }$ and (ii) the larger is the value of $\omega$ associated with $\sigma_{a}^{(l) \max }$. This is all compatible with the fact that the scattering potential $V_{a}$ is larger for larger values of $l$ (see Fig. 1).

In Fig. 3 we plot our results for the total absorption cross section $\sigma_{a}$. Our numerical result for $\sigma_{a}$ is in excellent agreement with the expected values both in the low- and high-frequency limits. There is a general result which guarantees that the absorption cross section of minimally coupled massless scalar fields for spherically symmetric black holes (in asymptotically flat spacetimes) equals the area of the black hole event horizon in the low-frequency regime [14]. Therefore, the absorption cross section in the zero-frequency limit should be

$$
\sigma_{a}^{l f}=4 \pi r_{H}^{2} .
$$

In the opposite limit, following Ref. [15] it can be easily shown that in the high-frequency (geometrical-optics) regime the absorption cross section is [16]

$$
\sigma_{a}^{h f}=(3 \sqrt{3} / 2) \pi r_{H}^{2} .
$$

Both Eqs. (15) and (16) can be explicitly verified in Fig. 3. It is also interesting to note that the absorption cross
PHYSICAL REVIEW D 76, 107502 (2007)

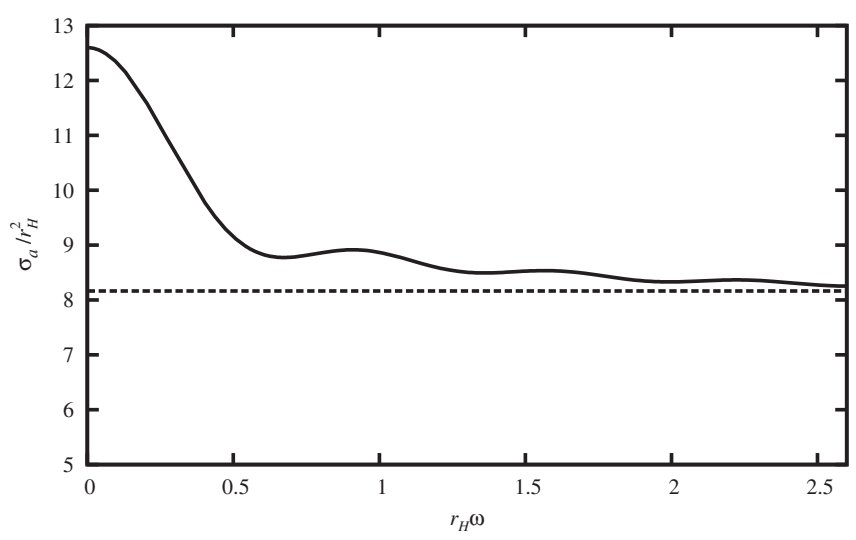

FIG. 3. The total absorption cross section $\sigma_{a}$ is plotted as a function of the wave frequency $\omega$. The summation in Eq. (14) is performed up to $l=6$. It starts equal to the black hole horizon area and falls approaching $\sigma_{a}^{h f}$ (see dashed line), in complete agreement with the results obtained in the low- and highfrequency approximations.

section for the canonical acoustic hole is smaller in the high-frequency regime than in the low-frequency one. In the case of a 4-dimensional Schwarzschild black hole we have just the opposite behavior for the massless scalar and electromagnetic fields (see Refs. [4,7], respectively). The total absorption cross section has local maxima corresponding to the maximum of each partial absorption cross section.

In summary we have numerically computed the absorption cross section of sound waves for a canonical acoustic hole for every frequency. Our results are in complete agreement with the expected low- and high-frequency limits.

The authors are grateful to Conselho Nacional de Desenvolvimento Científico e Tecnológico (CNPq) for partial financial support. E. O. and G. M. would like to acknowledge also partial financial support from Coordenação de Aperfeiçoamento de Pessoal de Nível Superior (CAPES) and Fundação de Amparo à Pesquisa do Estado de São Paulo (FAPESP), respectively. L.C. would like to thank the hospitality at the Abdus Salam International Centre for Theoretical Physics, where part of this work was developed.
[1] W. G. Unruh, Phys. Rev. Lett. 46, 1351 (1981).

[2] C. Barceló, S. Liberati, and M. Visser, Living Rev. Relativity 8, 12 (2005), www.livingreviews.org/lrr-200512.

[3] B. Mashhoon, Phys. Rev. D 7, 2807 (1973); R. Fabbri, Phys. Rev. D 12, 933 (1975); D. N. Page, Phys. Rev. D 13,
198 (1976); W. G. Unruh, Phys. Rev. D 14, 3251 (1976).

[4] N. Sanchez, Phys. Rev. D 18, 1030 (1978).

[5] L. C. B. Crispino, A. Higuchi, and G. E. A. Matsas, Phys. Rev. D 63, 124008 (2001).

[6] C. Doran, A. Lasenby, S. Dolan, and I. Hinder, Phys. Rev. D 71, 124020 (2005). 
[7] L. C. B. Crispino, E. S. Oliveira, A. Higuchi, and G. E. A. Matsas, Phys. Rev. D 75, 104012 (2007).

[8] M. Visser, Classical Quantum Gravity 15, 1767 (1998).

[9] N.D. Birrel and P.C.W. Davies, Quantum Fields in Curved Space (Cambridge University Press, Cambridge, England, 1982).

[10] M. Abramowitz and I. A. Stegun, Handbook of Mathematical Functions (Dover Publications, New York, 1965).

[11] S. Basak and P. Majumdar, Classical Quantum Gravity 20, 2929 (2003).
[12] I. S. Gradshteyn and I. M. Ryzhik, Tables of Integrals, Series, and Products (Academic Press, New York, 1980).

[13] L.C.B. Crispino, A. Higuchi, and G.E.A. Matsas, Classical Quantum Gravity 17, 19 (2000).

[14] S. R. Das, G. Gibbons, and S. D. Mathur, Phys. Rev. Lett. 78, 417 (1997).

[15] R. M. Wald, General Relativity (University of Chicago Press, Chicago, 1984).

[16] I. Zolnerkevic, Report No. IFT-M. 005/04 (available under request). 